\title{
Electromagnetic flow control in metallurgy, crystal growth and electrochemistry
}

\author{
Gunter Gerbeth $^{1, a}$, Kerstin Eckert ${ }^{2, b}$, and Stefan Odenbach ${ }^{2, c}$ \\ 1 Helmholtz-Zentrum Dresden-Rossendorf (HZDR), Institute of Fluid Dynamics, \\ 01314 Dresden, Germany \\ 2 Institute of Fluid Mechanics, Chair of Magnetofluiddynamics, Measuring and Automation \\ Technology, Technische Universität Dresden, 01069 Dresden, Germany
}

Electromagnetic fields, produced either by alternating (AC) or constant (DC) electric currents, or by simple permanent magnets, strongly influence the flow of liquid metals or electrolytes. This is caused by the Lorentz force (density) $\boldsymbol{f}_{L}=\boldsymbol{j} \times \boldsymbol{B}$, resulting from the interaction between the magnetic induction $\boldsymbol{B}$ and the electric current density $\boldsymbol{j}$, either induced by Faraday's law in liquid metals, or injected in the case of weakly conducting liquids, e.g. in electrochemical systems. The study of the feedback between $\boldsymbol{B}$ and the velocity field $\boldsymbol{u}$, driven by $\boldsymbol{f}_{L}$ is called magnetohydrodynamics (MHD), a discipline dating back to the discovery of the induction law in 1831 by Faraday. For the basics of MHD we refer to excellent textbooks [1-3] or a recent review [4]. Important early milestones in the development of MHD were the first liquid metal experiments in channels performed by Hartmann in 1937 [5] and the Nobel prize decorated discovery of incompressible waves in 1942 [6], now called Alfvén waves, that play an important role in solar physics. More recent developments consist in the rapid emergence of a new discipline, the electromagnetic processing of materials with own conferences [7], or the experimental proofs of the dynamo action, i.e. the self-excitation of $\boldsymbol{B}$ solely caused by the flowing liquid metal in specifically designed laboratory experiments [8].

This remarkable diversity of phenomena is sorted in MHD by the magnetic Reynolds number $R m=\mu_{0} \sigma u l$, where $\mu_{0}, \sigma, u$ and $l$ refer to vacuum permeability, electric conductivity and to the characteristic scales of velocity and length, respectively. The dynamo problem as well as other important astrophysical problems, such as phenomena in the solar atmosphere, fall into the class of high- $R m$ problems due to the large length scales involved. Here, the magnetic fields induced by the flow of electrically conducting liquids via the induction equation have to be taken into account, making the calculation of $\boldsymbol{f}_{L}$ a formidable task. By contrast, nearly all laboratory and industrial problems of MHD belong to the low- $R m$ range in which the acting $\boldsymbol{B}$ is the applied one, which drastically simplifies the determination of $\boldsymbol{f}_{L}$. This richness in topics on various length scales, including geo- and astrophysical problems as well

\footnotetext{
a e-mail: g.gerbeth@hzdr.de

b e-mail: Kerstin.Eckert@tu-dresden.de

c e-mail: Stefan.Odenbach@tu-dresden.de
} 
as the electromagnetic processing of material, makes MHD an attractive and important field of research. This is supported by the surprising breadth of liquid metal technologies which have recently evolved. They range from high-temperature energy conversion systems, the casting of steels and light-weight metals, liquid metal cooled systems or liquid metal targets in modern neutron sources, to new kinds of liquid metal batteries.

From 2002 to 2012 the German Science Foundation (DFG) has funded the Collaborative Research Center SFB 609, Electromagnetic flow control in metallurgy, crystal growth and electrochemistry. The SFB 609, represented by R. Grundmann (2001-2007) and S. Odenbach (2007-2012) combines 25 projects, several of which were performed through collaboration between different institutions. The TU Dresden hosted 14 subprojects, the Helmholtz-Zentrum Dresden-Rossendorf (HZDR) 9 projects, and the IFW Dresden and the TU Bergakademie Freiberg two projects each. The present volume gives a detailed overview of the research activities of SFB 609 in form of both minireviews and original research contributions. The volume is divided into five chapters corresponding to the main research topics of SFB609. The main content of the various contributions is briefly summarized in the following as a quick overview for the reader.

\section{Fundamentals}

The first contribution from Giesecke et al. [9] reviews SFB 609 activities devoted to high $R m$-problems. First, the two basic mathematical formulations, the differential equation approach, based on the induction equation and the integral equation approach that relies on Biot-Savart's law are discussed. Particular attention is paid to the correct treatment of the boundary conditions for the magnetic field. Both methods are applied to the French von-Kármán-Sodium dynamo experiment, for which the authors were able to show that the magnetic propeller materials strongly influence performance and mode selection.

The insights gained recently into the dynamo and numerous other problems are based on the tremendous progress which has been made in instrumentation and online monitoring of liquid metal flows. One of these new techniques, the Contactless Inductive Flow Tomography (CIFT), uses the magnetic field, measured in the exterior of the fluid, to infer the velocity field in the interior [9]. The state-of-the-art of two other prominent measuring techniques is reviewed in Cramer, Eckert and Gerbeth [10], namely electric potential difference measurements and ultrasound Doppler velocimetry. While the first allows for highly time-resolved local measurements, the latter provides velocity profiles along the propagation direction of the ultrasound waves. The potential of both measuring techniques, for turbulence measurements or to resolve the spatial structure of the flow, among other applications, is exemplified for a flow driven by a rotating magnetic field.

Nauber et al. [11] report on the recent progress in flow mapping by introducing a new multidimensional ultrasound Doppler velocimeter. It rests on multiple line arrays of transducers which allows to resolve small-scale structures with improved lateral and axial resolutions and frame rates up to $30 \mathrm{~Hz}$. The layout of the system including the advanced time division multiplexing scheme is explained. Selected experimental results are shown for rotating magnetic field (RMF)- and jet-driven flows.

The analysis of liquid-metal-gas two-phase flows, cf. Sec. 3, requires precise information on the gas bubble distribution in the liquid metal in addition to the measurements of the liquid metal velocity. This is made possible by means of an alternative technique, the ultrasound transit time technique. Andruszkiewicz et al. [12] discuss 
the operational principle and explain the extraction of, e.g., bubble positions, velocities or diameters from the ultrasound echograms. The performance of the technique is demonstrated in an argon-GaInSn flow in a static magnetic field.

X-ray attenuation techniques, reviewed in Shevchenko et al. [13], are another important diagnostic tool which has been established in the past decade. X-ray visualization enables a general intuitive understanding of flow phenomena or pattern formation in opaque liquid metal systems. The authors demonstrate how real-time and in-situ observations of the density distribution in thin solidifying samples with a spatial resolution of a few microns have facilitated an improved understanding of dendritic growth processes. Moreover, they show that the X-ray radioscopy is a useful tool for non-invasive, in-situ visualization and characterization of gas bubbles in nontransparent melts or for observations of the formation of metal foams.

Besides liquid metals, SFB 609 has also focused on weakly electrically conducting liquids. König et al. [14] discuss an advanced laser Doppler profile sensor for spatially resolved measurements in such liquids. Using two fan-like interference systems, instead of the constant fringes spacing of the classical laser Doppler anemometer, their sensor is able to measure velocity profiles. This is particularly useful to resolve details of convective flows in close proximity to the electrode in electrochemical systems as demonstrated in two examples.

In contrast to the these measurement techniques, Albrecht et al. [15] discuss numerical approaches to derive force densities from a velocity field, measured, e.g., by particle image velocimetry. They first compare the accuracy of different methods in reconstructing the required pressure distribution and determine which methods are best suited for computing either drag or lift. In the second part, solutions are given for the force density that a plasma actuator, whose physical operation principle is still a matter of debate, exerts on a fluid flow.

Liquid metal suspensions with solid particles are a technologically relevant class, the rheological behavior of which is presently only poorly understood. A mandatory step for better characterization is the defined incorporation of particles into the liquid metal. Borin and Odenbach [16] evaluate different methods of particle injection, such as mechanical and electromagnetic stirring, laser injection and metallurgical sintering. In particular, the oscillating cup technique was used to measure the viscosity of selected metal suspensions, such as liquid $\mathrm{Sn}$ with varying concentration of $\mathrm{SnO}_{2}$ particles. These results are presented.

\section{Electromagnetic flow control in casting and solidification}

Magnetic fields are applied in the casting industry either as DC fields in electromagnetic brakes in the continous casting of steel or as AC fields for electromagnetic stirring. Electromagnetic brakes are expected to damp turbulent fluctuations, thereby avoiding undesirable slag entrainment from the surface into the mold. Electromagnetic stirring, applied to solidifying melts, is able to produce strongly refined microstructures. Although studies to understand the flow fields produced by electromagnetic stirring date back to the 1980s, significant progress has been made in the past ten years through a combination of model experiments, the use of low-melting-point alloys and adapted numerical simulations.

Stiller et al. [17] review the current understanding of flows driven by rotating (RMF) and traveling (TMF) magnetic fields as achieved by three-dimensional direct numerical and large-eddy simulations and matched model experiments. Furthermore, the flow fields resulting from superpositions of RMFs and TMFs are considered. The authors point out that TMF-driven flows show a pronounced sensitivity to misalignment between the axes of the cylindrical liquid metal column and the TMF. Even a 
$1 \mathrm{~mm}$ radial shift between the axes is able to eliminate the well-known toroidal flow structure.

Eckert et al. [18] provide an overview of the impact of RMF- and TMF-stirring on both the macro- and microstructure of solidifying alloys. Flow-induced effects such as modifications in the grain orientation, earlier onset of the columnar to equiaxedtransition or grain refinement and dendrite fragmentation are discussed in detail. The origin of freckle formation is substantiated based on real-time X-ray observation experiments. Based on numerical simulations it is shown that continuous stirring in RMFs leads to characteristic fir-tree like concentration inhomogeneities along the axis, the shape of which is a clear fingerprint of Taylor-Görtler vortices, which appear at sufficiently high Taylor numbers in the melt and are dissipated in the mushy zone. A new strategy to overcome such macrosegregations by means of temporally modulated magnetic fields is outlined.

Casting of light-weight aluminum alloys in a TMF is studied by Metan and Eigenfeld [19] with and without grain refining particles. Both electromagnetic stirring and addition of grain refiners significantly reduce the mean grain size. The use of grain refining particles appeared to be more effective for the alloys selected. In parallel, the resulting mechanical strength and the electric conductivity of the alloy produced is measured. It appears that the grain refiners become less effective with progressing solidification since a coarsening of the mean grain area was observed along the horizontal axis of the samples. While TMFs are of particular use in alloys that do not allow the use of grain refining particles, TMFs are even helpful in the presence of such grain refining particles to achieve good mixing of the particles by counteracting sedimentation.

Timmel et al. [20] analyze the current understanding of the mold flow in the continuous casting of steel in the presence of DC magnetic fields. While various types of magnetic systems are already in industrial use, the actual impact on the melt flow in this process is only partly elucidated. A significant advancement has been achieved at HZDR with the construction of three experimental facilities providing Liquid Metal Models for the continuous CASTing of steel (LIMMCAST) which operate with the low-melting alloys GaInSn and $\mathrm{SnBi}$, respectively. The authors discuss recent results obtained by the combined application of different measurement techniques. Local potential probes, ultrasound Doppler Velocimetry (UDV) and CIFT are applied for measurements of the melt flow while Mutual Inductance Tomography and X-ray radioscopy are employed to study the argon-liquid-metal flow behavior both in the submerged entry nozzle and in the mold. Numerical calculations using ANSYS-CFX with an implemented RANS-SST turbulence model complement the study.

\section{Liquid metal two-phase flows}

Many technical applications in power engineering, metallurgy and casting rely on liquid-metal two-phase flows. For instance, gas injection is applied routinely at various stages to promote chemical reactions or to stir the melt to achieve better homogenization. Magnetic fields enable effective and contactless control of the bubble motion in the liquid metal, thereby offering the possibility to enhance the mixing rate.

Fröhlich et al. [21] review numerical and experimental investigations devoted to the physics of rising bubbles in conducting liquid metals under the action of various magnetic fields. Different bubble regimes, from single bubbles to bubble chains toward bubble plumes and bubbles swarms, are considered. The induced Lorentz forces act on the non-conducting bubbles by altering their wakes, as demonstrated by direct numerical simulations. These small-scale effects modify the trajectory of the ascending bubbles such that the bubble follow a straighter path than in the absence of a magnetic 
field. Selected results are presented from the new generation of measuring techniques, including X-ray radioscopy and ultrasound techniques, from which further detailed experimental data on an unprecedented scale can be expected. Beside advancing our general understanding of this flow class these data are particularly meaningful for the validation of numerical codes.

The latter topic forms the focus of the study by Aland et al. [22] in which different numerical approaches to simulate the rise of a single bubble are compared. The first one is a simplified model which assumes that the bubble as solid-like, motivated by the agglomeration of inclusions at the interface, and employs an immersed boundary method (IBM). Ellipsoidal shape deformations, depending on the bubble Weber number are incorporated. The second approach is a Navier-Stokes-Cahn-Hilliard (NSCH) model which considers the two-phase problem and takes surface tension and the jump in the boundary conditions into account. Depending on the surface tension and the viscosity ratio, the authors identify parameter regions of high correspondence of both models. This opens a route for large-scale simulations with high accuracy using highly simplified models.

An IBM method is also used by Schwarz and Fröhlich [23] for three-dimensional simulations of bubble chains in a static magnetic field directed parallel to the gravity vector. Bubble-bubble and bubble-wall interactions, which occur during the bubble rise in the narrow container, are modelled by means of a repelling potential. In the presence of the magnetic field, the rise of the bubbles proceeds with a smaller average velocity and on more rectilinear trajectories. In parallel, the overall circulation in the container is reduced.

Finally, Heitkam et al. [24] simulate large bubble ensembles in the form of wet metal foams. To control the bubbles they inject a horizontal electric current in the presence of an orthogonal static magnetic field, generating an upwards directed Lorentz force. At a critical value of the latter, compensation of the gravity field is achieved and the bubbles start to float, thereby filling the upper part of the computational domain. Due to the non-vanishing curl of the Lorentz force density, the floating bubbles are not stationary but mobile. Such a configuration allows manipulation of the bubble distribution during foam generation. Simple calculations show realistic opportunities for real metal foams.

\section{Application of magnetic fields in crystal growth technologies}

Vertical Bridgman/Gradient Freeze (VB/VGF) type growth, the Czochralski $(\mathrm{Cz})$ process and floating zone single crystal growth are the dominating techniques through which the majority of semiconductor materials is produced worldwide. Although different in technical realization, all three processes have in common that the transport processes in the melt significantly influence the properties of the crystals grown. Thus, flow control by magnetic fields became an important issue for both the quality of the crystal and the economic aspect of its production.

Hence, the questions of flow stability as addressed by Grants, Galindo and Gerbeth [25] for the VGF and $\mathrm{Cz}$ processes are crucial. Among other differences, the processes produce different thermal stratifications, unstable for $\mathrm{Cz}$ but stable for VGF growth. The authors show for the VGF case that the stability of RMFdriven flows is, counterintuitively, decreased by the stable stratification. By contrast, TMF-driven flows experience a stabilization. For the thermally unstable configuration in the $\mathrm{Cz}$ process, the RMF in turn creates a distinct transition from a turbulent, large-scale, low-frequency convection to a small-scale, high-frequency, turbulent flow. This transition could be the essential basis for the use of a higher initial melt filling level in the $\mathrm{Cz}$ process. 
The contribution of Hermann, Gerbeth and Priede [26] is devoted to radiofrequency floating zone growth of single crystals of intermetallic compounds. The floating-zone technique is generally suitable for semiconductor materials with highmelting points since it allows for crucible-free growth. However, the convection driven by the inductive heating of the melt is often responsible for an undesirable curvature of the solid-liquid interface, which leads to poly- instead of single crystalline growth. The authors explain the principle of their two-phase stirrer, invented in this project, which changes the standard double vortex into a single roll structure. In this way, the desired interface curvature can be adjusted, enabling single crystal growth over the entire cross section which was recently successfully applied to antiferromagnetic Heusler compounds and biocompatible titanium alloys.

Pätzold et al. [27] review the VB/VGF-type growth under the influence of different magnetic fields, including rotating, travelling, and static fields. The authors show that the radial segregation of the dopant distribution is significantly reduced by an RMF while a downwards directed TMF decreases concave interface deflection, facilitating the desired shear stress reduction. Of particular relevance are also the results obtained for the superposition of RMF and TMF with static axial DC fields. Here, flow stabilization is achieved resulting in a partial or complete suppression of striation patterns.

Cramer, Pal and Gerbeth [28] report on model experiments for the $\mathrm{Cz}$ process which go beyond the widely studied Rayleigh-Bénard case. Two sophisticated experimental set-ups were created to provide experimental data relevant for the real-scale $\mathrm{Cz}$ process. The flow is dominated by a non-axisymmetric large-scale wind as demonstrated by detailed velocity and temperature measurements.

It is also noteworthy that a so-called SFB transfer project resulted out of the basic, crystal growth oriented studies of this section. In that project, HZDR and TU Bergakademie Freiberg along with an industry partner address the problem of optimized flows for the growth of multi-crystalline silicon for photovoltaic applications. Special attention is given to the influence of the flow on the possible agglomeration of impurities that result in defects in the solidified ingot. As this project is still active through mid-2014, no detailed report is given in the present volume.

\section{Flow control in electrolytes and magneto-electrochemistry}

In addition to liquid metal and semiconductor melts, weakly electrically conducting liquids, such as electrolytes, form the third class of fluids studied in the SFB 609 . Such liquids are employed throughout the field of electrochemistry. Transport of the electroactive ions in electrochemical systems proceeds via three processes: diffusion, migration in the electric field and convection. Convection can be easily forced with even small permanent neodymium-iron-boron magnets both by the Lorentz force, and on a smaller scale by the magnetic field gradient force. The resulting enhanced convective transport may significantly improve the quality of the functional layers deposited, e.g. by decreasing grain size and increasing the homogeneity of their thickness. Furthermore it may increase the yield of gas-evolving processes which are of tremendous importance in the electrochemical industry.

Before coming to these magnetoelectrochemical studies, the section starts with another aspect of flow in electrolytes, as outlined in the contribution by Albrecht et al. [29], namely flow control by means of Lorentz force actuators. Consisting of alternating combinations of electrodes and magnets, these actuators generate Lorentz forces in fluid layers close to the walls of the container. In this way, momentum can be transferred locally into the boundary layer at adjustable amplitude and penetration depth. The authors review recent achievements in drag reduction in turbulent 
boundary layers and in delaying the transition to turbulence. Finally, aspects of the control of flow separation around inclined airfoils are addressed. Although the Lorentz force actuator can control a wide range of flow phenomena, its applicability is still constrained to small- and medium-scale systems due to low electrical-to-mechanical efficiency.

Electrochemical deposition, as a widely used and economic technique for surface finishing, is extended by Uhlemann et al. [30] to achieve structured electrodeposition in magnetic field gradients. Prerequisite for this process is the existence of paramagnetic species, since only those are affected by the magnetic gradient force. The Lorentz force plays only a minor role here. Three different modes of structuring could be successfully established, (i) direct structuring for strongly paramagnetic ions, (ii) reverse structuring when the electroactive species are diamagnetic and (iii) the formation of free-standing structures in pulse-reverse plating. By reviewing the existing experimental approaches and the numerical simulations performed, the current understanding of the process is summarized by showing that locally induced convection plays a key role in the structuring.

Electrodeposition is frequently accompanied by natural convection which may lead, on a longer time-scale, to an undesirable stable density stratification inside the cell. Previous works of the SFB [31] therefore favor a transient operation. Utilizing a combination of particle image velocimetry and Mach-Zehnder interferometry, Yang et al. [32] show that the originally smooth start-up of pure natural convection is converted into a highly unsteady process in the presence of an opposing Lorentz force. The reason is the advection of the less-concentrated cupric ion solution in a bubble-like zone of high buoyancy at the lower part of the vertical cathode when copper electrolysis is performed. Despite the Lorentz-force-driven counterflow, the time at which buoyancy-driven convection is fully established scales with the Rayleighnumber dependence of the pure buoyancy-driven case which is a surprising result.

The two-phase flow originating from alkaline water electrolysis at a gas-evolving electrode is studied by Weier and Landgraf [33] by a combination of particle image velocimetry, fluorescent tracers, and high-speed imaging of bubble shadows. They show that the boundary of the hydrogen bubble curtain at the cathode is wavy because the wall-normal velocity component oscillates with a frequency in the Hertz range. In experiments with permanent magnets mounted behind the cathode such that the Lorentz force supports the bubble-driven convection, the authors show an enormous increase in the fluid velocity. This indicates the potential of Lorentz forces for gasevolving electrodes and encourages further study in this area.

With this overview we hope to have convinced the reader that MHD is an active and vibrant discipline. We are lucky that Germany's Helmholtz Association is supporting a new large-scale program, called the Helmholtz Alliance for Liquid Metal Technologies (LIMTECH), which extends the work of SFB 609 in new directions.

We thank Deutsche Forschungsgemeinschaft (DFG) for the financial support of the Collaborative Research Center (SFB) 609, Electromagnetic Flow control in metallurgy, crystal growth and electrochemistry. Furthermore we express our thanks to Sabine Lehr and Chris Caron, Springer Verlag, for the pleasant collaboration.

\section{References}

1. J. Shercliff, Textbook of Magnetohydrodynamics (Pergamon Press, New York, 1965)

2. R. Moreau, Magnetohydrodynamics, vol. 3 (Springer, 1990)

3. P. Davidson, An Introduction to Magnetohydrodynamics, vol. 25 (Cambridge University Press, 2001) 
4. S. Molokov, R. Moreau, H.K. Moffatt (eds.), Magnetohydrodynamics: Historical Evolution and Trends, vol. 80 (Springer, 2007)

5. J. Hartmann, F. Lazarus, Det Kgl. Danske Videnskabernes Selskab. Math.-Fys. Meddelelser 15, 1 (1937)

6. H. Alfvén, Nature 150, 405 (1942)

7. Proceedings of the Int. Conference EPM2012, Beijing, J. Iron Steel Research 19, Supplements 1-1 and 1-2 (2012)

8. F. Stefani, A. Gailitis, G. Gerbeth, ZAMM-Journal Appl. Math. Mechanics/Zeitschrift Angewandte Math. Mech. 88, 930 (2008)

9. A. Giesecke, F. Stefani, T. Wondrak, M. Xu, Eur. Phys. J. Special Topics 220, 9 (2013)

10. A. Cramer, S. Eckert, G. Gerbeth, Eur. Phys. J. Special Topics 220, 25 (2013)

11. R. Nauber, M. Burger, L. Büttner, S. Franke, D. Räbiger, S. Eckert, J. Czarske, Eur. Phys. J. Special Topics 220, 43 (2013)

12. A. Andruszkiewicz, K. Eckert, S. Eckert, S. Odenbach, Eur. Phys. J. Special Topics 220, $53(2013)$

13. N. Shevchenko, S. Boden, S. Eckert, D. Borin, M. Heinze, S. Odenbach, Eur. Phys. J. Special Topics 220, 63 (2013)

14. J. König, M. Neumann, S. Mühlenhoff, K. Tschulik, T. Albrecht, K. Eckert, M. Uhlemann, T. Weier, L. Büttner, J. Czarske, Eur. Phys. J. Special Topics 220, $79(2013)$

15. T. Albrecht, V. del Campo, T. Weier, H. Metzkes, J. Stiller, Eur. Phys. J. Special Topics 220, 91 (2013)

16. D. Borin, S. Odenbach, Eur. Phys. J. Special Topics 220, 101 (2013)

17. J. Stiller, K. Koal, W. Nagel, J. Pal, A. Cramer, Eur. Phys. J. Special Topics 220, 111 (2013)

18. S. Eckert, P. Nikrityuk, B. Willers, D. Räbiger, N. Shevchenko, H. Neumann-Heyme, V. Travnikov, S. Odenbach, A. Voigt, K. Eckert, Eur. Phys. J. Special Topics 220, 123 (2013)

19. V. Metan, K. Eigenfeld, Eur. Phys. J. Special Topics 220, 139 (2013)

20. K. Timmel, X. Miao, T. Wondrak, F. Stefani, D. Lucas, S. Eckert, G. Gerbeth, Eur. Phys. J. Special Topics 220, 151 (2013)

21. J. Fröhlich, S. Schwarz, S. Heitkam, C. Santarelli, C. Zhang, T. Vogt, S. Boden, A. Andruszkiewicz, K. Eckert, S. Odenbach, S. Eckert, Eur. Phys. J. Special Topics 220, 167 (2013)

22. S. Aland, S. Schwarz, J. Fröhlich, A. Voigt, Eur. Phys. J. Special Topics 220, 185 (2013)

23. S. Schwarz, J. Fröhlich, Eur. Phys. J. Special Topics 220, 195 (2013)

24. S. Heitkam, S. Schwarz, C. Santarelli, J. Fröhlich, Eur. Phys. J. Special Topics 220, 207 (2013)

25. I. Grants, V. Galindo, G. Gerbeth, Eur. Phys. J. Special Topics 220, 215 (2013)

26. R. Hermann, G. Gerbeth, J. Priede, Eur. Phys. J. Special Topics 220, 227 (2013)

27. O. Pätzold, K. Niemietz, R. Lantzsch, V. Galindo, I. Grants, M. Bellmann, G. Gerbeth, Eur. Phys. J. Special Topics 220, 243 (2013)

28. A. Cramer, J. Pal, G. Gerbeth, Eur. Phys. J. Special Topics 220, 259 (2013)

29. T. Albrecht, J. Stiller, H. Metzkes, T. Weier, G. Gerbeth, Eur. Phys. J. Special Topics 220, 275 (2013)

30. M. Uhlemann, K. Tschulik, A. Gebert, G. Mutschke, J. Fröhlich, A. Bund, X. Yang, K. Eckert, Eur. Phys. J. Special Topics 220, 287 (2013)

31. X. Yang, K. Eckert, S. Mühlenhoff, S. Odenbach, Electrochimica Acta 54, 7056 (2009)

32. X. Yang, S. Mühlenhoff, P. Nikrityuk, K. Eckert, Eur. Phys. J. Special Topics 220, 303 (2013)

33. T. Weier, S. Landgraf, Eur. Phys. J. Special Topics 220, 313 (2013) 